\title{
The Frontiers of Catalysis Science and Future Challenges
}

\author{
Hans-Joachim Freund • Gabor A. Somorjai
}

(C) Springer Science+Business Media New York 2014

In the 10th Anniversary issue of Catalysis Letters in 2000, Professor M. W. Roberts reviewed the history of catalysis. This 25th Anniversary of the journal is a major milestone, and thus we have compiled an issue that has papers from some of the leading contributors to the frontiers of catalysis.

We believe that now, on the occasion of the Silver Anniversary of the publication of Catalysis Letters, it is appropriate to focus on the rapid growth of the field, its development into molecular catalysis science and the future challenges that will be presented in the next 25 years. The importance of catalysis in energy conversion, in environmental remediation and in the development of new pharmaceuticals and biological chemistry has assured exponential development of the field equally in heterogeneous, homogeneous and enzyme catalysis over the past 25 years. While catalysis science used to be published in specialty journals, it has now entered the mainstream; in addition, the number of journals that publish papers with content chiefly dealing with catalysis has multiplied. Catalysis research is carried out and growing now on all of the continents with particular emphasis on Europe, the Americas, and Asia. The past 25 years have seen a transition of the field from one dominated by catalytic technologies to molecular catalysis science.

H.-J. Freund $(\square)$

Department of Chemical Physics, Fritz-Haber-Institut der MaxPlanck-Gesellschaft, Faradayweg 4-6, 14195 Berlin, Germany

e-mail: topcatlett@fritz-haber-institute.de

G. A. Somorjai

Department of Chemistry, University of California, Berkeley, CA 94720-1460, USA

e-mail: somorjai@berkeley.edu
There are three areas of catalysis science in which major advances have been made.

1. With the rise of nanomaterial science, the synthetic methodologies to fabricate catalysts underwent a revolution, since all catalysts are nanoparticles in the size range of 1-10 nm. Colloid chemistry, organometallic complexes and clever methods for producing enzymes are the ways by which catalysts are produced in large quantities

2. Atomic and molecular level characterization have been extended by using many new photon based techniques to monitor dynamic changes to well-structured model systems under reaction conditions. Parameters measured include structure, composition, oxidation state, and mobility during bond formation and bond scission with spatial and time resolutions

3. Catalytic reactor technologies, including miniaturized reactors, that are used to investigate transient processes occurring in the $10^{-3}$ to $10 \mathrm{~s}$ range permit us to obtain kinetic information about the transition states that accompany chemical changes and thus to extend our reach beyond the steady state. Those studies are carried out using a combination of advanced new instruments in combination with novel theoretical approaches. Increasingly, complex catalyst architectures carry out seamless multistep catalytic processes in novel catalytic environments to bridge the gap between homogeneous and heterogeneous catalysis

The aim of modern catalytic studies is to obtain desired products and reactants with $100 \%$ selectivity in order to optimize product yield and eliminate wasteful byproducts. Clearer and more detailed understanding of catalytic science is required to achieve the goals of producing clean energy, clean air, and clean water, and discovering new 
drugs to increase life expectancy. If we understand heterogeneous, homogeneous, and enzyme catalysis on the molecular level, new discoveries at the boundaries of these three fields will provide the knowledge for the construction of hybrid catalysis and thus further expansion of molecular catalysis science. Interdisciplinary knowledge of solid-state physics, chemistry, biology, and medicine, coupled with frontier engineering are needed to discover new catalysis science and develop new catalyst-based technologies. Increasingly, catalysis and its applications will become the frontier area of study in many fields that ultimately control and improve our quality of life.

We would like to thank all the contributors to this Silver Anniversary issue and all the many researchers who have contributed to the success of Catalysis Letters and Topics of Catalysis over the years. 